\title{
Practical and Highly Enantioselective Synthesis of $\beta$-Alkynyl- $\beta$-Amino Esters Through Ag-Catalyzed Asymmetric Mannich Reactions of Silylketene Acetals and Alkynyl Imines
}

\author{
Nathan S. Josephsohn, Emma L. Carswell, Marc L. Snapper* and Amir H. Hoveyda* \\ Department of Chemistry, Merkert Chemistry Center, Boston College \\ Chestnut Hill, Massachusetts 02467
}

\section{SUPPORTING INFORMATION \\ PART 1}

General. Infrared (IR) spectra were recorded on a Perkin Elmer 781 spectrophotometer, $v_{\max }$ in $\mathrm{cm}^{-1}$. Bands are characterized as broad (br), strong (s), medium (m), or weak (w). ${ }^{1} \mathrm{H}$ NMR spectra are recorded on a Varian Unity INOVA $400(400 \mathrm{MHz})$ spectrometer. Chemical shifts are reported in ppm from tetramethylsilane with the solvent resonance resulting from incomplete deuteration as the internal standard $\left(\mathrm{CDCl}_{3}: \delta 7.26\right)$. Data are reported as follows: chemical shift, integration, multiplicity $(\mathrm{s}=$ singlet, $\mathrm{d}=$ doublet, $\mathrm{t}=$ triplet, $\mathrm{q}=$ quartet, $\mathrm{br}=$ broad, $\mathrm{m}=$ multiplet), and coupling constants in $\mathrm{Hz} .{ }^{13} \mathrm{C}$ NMR spectra were recorded on a Varian Unity INOVA $400(100 \mathrm{MHz})$ with complete proton decoupling. Chemical shifts are reported in ppm from tetramethylsilane with the solvent resonance as the internal standard $\left(\mathrm{CDCl}_{3}: \delta 77.16\right)$. Enantiomeric ratios were determined by high-performance liquid chromatography (HPLC) on a Shimadzu chromatograph and chiralpak AD, OD and OJ columns (4.6 mm x $250 \mathrm{~mm}$ chiral column by Chiral Technologies) in comparison with authentic racemic materials. All highresolution mass spectrometry were performed at Boston College on a Micromass LCT ESI-MS (positive mode). Optical rotation values were recorded on a Rudolph Research Analytical Autopol IV polarimeter.

Unless otherwise stated, all reactions conducted under open atmosphere in $13 \mathrm{x} 100 \mathrm{~mm}$ borosilicate test tubes. J.T. Baker reagent grade THF was used without purification. Fisherbrand HPLC grade 2-Propanol was used without purification. Unless otherwise stated, silver acetate (99\%), iodobenzene diacetate, $\operatorname{Pd}(\mathrm{C})$, phenylpropargyl aldehyde, succinic anhydride, 4aminobenzamidine dihydrochloride, DMF, $p$-anisidine and $o$-anisidine were purchased from Aldrich and used without purification. Unless otherwise noted, $\mathrm{EDC} \cdot \mathrm{HCl}, \mathrm{HOBt} \cdot \mathrm{H}_{2} \mathrm{O}, \mathrm{Boc}_{2} \mathrm{O}$, Cbz-Val-OSu were purchased from Advanced Chemtech and used without purification. All alkynyl imines, ${ }^{1}$ silylketene acetal $\mathbf{3}^{2}$ and ligand $\mathbf{1}^{3}$ were prepared according to previously reported procedures.

1) Journet, M.; Cai, D.; DiMechele, L. M.; Larson, R. D. Tetrahedron Lett. 1998, 39, 6427-6428.

2) Colvin, E. W. Silicon Reagents in Organic Synthesis, Academic Press, Boston 1988. 
Representative procedure for Ag-catalyzed asymmetric Mannich reaction with silylketene acetals. Chiral phosphine $1(10.0 \mathrm{mg}, 20 \mu \mathrm{mol})$ and AgOAc (3.3 mg, $20 \mu \mathrm{mol})$ were weighed into a $13 \times 100 \mathrm{~mm}$ test tube. The contents were dissolved in $0.5 \mathrm{~mL}$ of THF and allowed to stir for $5 \mathrm{~min}$ at $22^{\circ} \mathrm{C}$. Imine $2 \mathbf{b}(94 \mathrm{mg}, 0.4 \mathrm{mmol})$ was added immediately followed by $35 \mu \mathrm{L} i$-PrOH $(0.45 \mathrm{mmol})$. The test tube was capped with a septum, and allowed to stir in a cryocool bath at $-60{ }^{\circ} \mathrm{C}$ for $5 \mathrm{~min}$. At this point, silyl ketene acetal 3 (167 $\mathrm{mg}, 0.8 \mathrm{mmol}$ ) was added and the resulting mixture was allowed to stir at $-60{ }^{\circ} \mathrm{C}$ for $16 \mathrm{~h}$. The reaction was quenched upon addition of $0.40 \mathrm{~mL}$ of a saturated aqueous solution of $\mathrm{NaHCO}_{3}$ at $-60{ }^{\circ} \mathrm{C}$ before allowing to warm to room temperature with vigorous stirring for $10 \mathrm{~min}$. The reaction mixture was then filtered through silica gel and subsequently washed with 1:1 hexanes:Et ${ }_{2} \mathrm{O}(150 \mathrm{~mL})$. The filtrate was concentrated and the resulting crude brown oil purified by silica gel chromatography $\left(9: 1\right.$ hexanes: $\left.\mathrm{Et}_{2} \mathrm{O}\right)$ to deliver $135 \mathrm{mg}(0.36 \mathrm{mmol}, 91 \%$ yield $)$ of the desired product $\mathbf{4 b}$ as a light yellow oil.

(+)-3-(2-Methoxy-phenylamino)-5-trimethylsilanyl-pent-4-ynoic acid phenyl ester (4a). Isolated as a light yellow oil. IR (neat, $\mathrm{NaCl}$ ): 3395 (br), 2974 (w), 2369 (w), 1759 (s), 1602 (m), 1507 (s), 1457 (m), 1255 (s), 1224 (m), 1199 (m), 1161 (m), 1130 (m), 1029 (w), 846 (s), 746 (m), $683(\mathrm{w}) .{ }^{1} \mathrm{H} \mathrm{NMR}\left(\mathrm{CDCl}_{3}, 400 \mathrm{MHz}\right): \delta$ 7.35-7.30 $(2 \mathrm{H}, \mathrm{m}), 7.21-7.16(1 \mathrm{H}, \mathrm{m}), 7.05-7.02$ $(2 \mathrm{H}, \mathrm{m}), 6.87-6.70(4 \mathrm{H}, \mathrm{m}), 4.71-4.65(2 \mathrm{H}, \mathrm{m}), 3.80(3 \mathrm{H}, \mathrm{s}), 3.06(1 \mathrm{H}, \mathrm{dd}, J=15.0,6.0), 2.96$ $(1 \mathrm{H}, \mathrm{dd}, J=15.0,6.2), 0.09(9 \mathrm{H}, \mathrm{s}) .{ }^{13} \mathrm{C} \mathrm{NMR}\left(\mathrm{CDCl}_{3}, 100 \mathrm{MHz}\right): \delta 169.0,150.7,147.8,135.9$, $129.5,126.1,121.7,121.3,118.4,112.3,110.0,104.6,88.5,55.7,43.5,41.0,0.07$. HRMS (ES) Calcd for $\mathrm{C}_{21} \mathrm{H}_{25} \mathrm{NO}_{3} \mathrm{NaSi}$ : 390.1488; Found: $390.1501(\mathrm{M}+\mathrm{Na}) . \quad[\alpha]_{\mathrm{D}}{ }^{20}=+50.5^{\circ}(c=0.867$, $\mathrm{CHCl}_{3}$ ) for a $92 \%$ ee sample.

Optical purity of 4a was established by chiral HPLC analysis (OD column, 90/10 hexanes/iPrOH eluent, $1 \mathrm{~mL} / \mathrm{min}$, lamp setting of $230 \mathrm{~nm}, \mathrm{R}_{\mathrm{t}}($ major $)=9.45, \mathrm{R}_{\mathrm{t}}($ minor $\left.)=15.57\right)$.

(+)-3-(2-Methoxy-phenylamino)-5-phenyl-pent-4-ynoic acid phenyl ester (4b). Isolated as a light yellow oil. IR (neat, $\mathrm{NaCl}$ ): 3389 (s), 1759 (s), 1602 (m), 1501 (s), 1457 (m), 1350 (m), 1249 (s), 1224 (s), 1186 (s), 1167 (s), 1123 (s), 1029 (m), 746 (s), 695 (s). ${ }^{1} \mathrm{H} \mathrm{NMR} \mathrm{(CDCl} 3,400$ $\mathrm{MHz}): \delta$ 7.41-7.35 (4H, m), 7.31-7.22 (4H, m), 7.13-7.10 (2H, m), 6.95-6.92 (2H, m), 6.84-6.77 $(2 \mathrm{H}, \mathrm{m}), 5.00-4.95(1 \mathrm{H}, \mathrm{bm}), 4.86-4.84(1 \mathrm{H}, \mathrm{bm}), 3.86(3 \mathrm{H}, \mathrm{s}), 3.22(1 \mathrm{H}, \mathrm{dd}, J=15.1,6.8), 3.15$ $(1 \mathrm{H}, \mathrm{dd}, J=15.1,6.6) .{ }^{13} \mathrm{C} \mathrm{NMR}\left(\mathrm{CDCl}_{3}, 100 \mathrm{MHz}\right): \delta 169.1,150.7,147.8,135.9,131.9,129.6$, $128.5,128.3,126.1,122.7,121.7,121.3,118.4,112.2,110.0,88.2,83.7,55.6,43.3,41.1$. HRMS (ES) Calcd for $\mathrm{C}_{24} \mathrm{H}_{21} \mathrm{NO}_{3} \mathrm{Na}$ : 394.1425; Found: $394.1419(\mathrm{M}+\mathrm{Na})$. $[\alpha]_{\mathrm{D}}{ }^{20}=+54.7^{\circ}(c=$ $3.03, \mathrm{CHCl}_{3}$ ) for a $92 \%$ ee sample.

Optical purity of $\mathbf{4 b}$ was established by chiral HPLC analysis (OD column, 92/8 hexanes/ $i$ PrOH eluent, $1 \mathrm{~mL} / \mathrm{min}$, lamp setting of $254 \mathrm{nmm}, \mathrm{R}_{\mathrm{t}}($ major $)=27.81, \mathrm{R}_{\mathrm{t}}($ minor $\left.)=30.64\right)$.

3) Josephsohn, N. J.; Snapper, M. L.; Hoveyda, A. H. J. Am. Chem. Soc. 2003, 125, 1418-1419. 
(+)-5-(4-Methoxy-phenyl)-3-(2-methoxy-phenylamino)-pent-4-ynoic acid phenyl ester (4c). The representative procedure described above was followed, except that reaction time was 36 h. 4c was isolated as a light yellow oil. IR (neat, NaCl): 3389 (w), 2835 (w), 1759 (s), 1608 (s), 1513 (s), 1451 (m), 1249 (s), 1199 (m), 1167 (m), 1029 (m), 840 (m), 739 (m), 689 (m). ${ }^{1} \mathrm{H}$ NMR $\left(\mathrm{CDCl}_{3}, 400 \mathrm{MHz}\right): \delta$ 7.38-7.30 $(4 \mathrm{H}, \mathrm{m}), 7.25-7.20(1 \mathrm{H}, \mathrm{m}), 7.10-7.08(2 \mathrm{H}, \mathrm{m}), 6.93-6.90$ $(2 \mathrm{H}, \mathrm{m}), 6.93-6.75(4 \mathrm{H}, \mathrm{m}), 4.95(1 \mathrm{H}, \mathrm{dd}, J=6.8,6.6), 4.83(1 \mathrm{H}, \mathrm{bs}), 3.85(3 \mathrm{H}, \mathrm{s}), 3.78(3 \mathrm{H}, \mathrm{s})$,

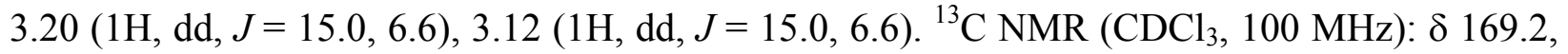
$159.7,150.7,147.7,136.0,133.3,129.5,126.1,121.7,121.3,118.4,114.8,113.9,112.2,110.0$, 86.7, 83.6, 55.6, 55.4, 43.3, 41.2. HRMS (ES) Calcd for $\mathrm{C}_{25} \mathrm{H}_{24} \mathrm{NO}_{4}$ : 402.1701; Found: $402.1705\left(\mathrm{M}+\mathrm{H}^{+}\right) \cdot[\alpha]_{\mathrm{D}}{ }^{20}=+52.8^{\circ}\left(c=2.00, \mathrm{CHCl}_{3}\right)$ for a $92 \%$ ee sample.

Optical purity of $\mathbf{4 c}$ was established by chiral HPLC analysis (OD column, 80/20 hexanes/ $i$ PrOH eluent, $1 \mathrm{~mL} / \mathrm{min}$, lamp setting of $254 \mathrm{~nm}, \mathrm{R}_{\mathrm{t}}($ major $)=18.78, \mathrm{R}_{\mathrm{t}}($ minor $\left.)=27.70\right)$.

3-(2-Methoxy-phenylamino)-5-naphthalen-1-yl-pent-4-ynoic acid phenyl ester (4d). The representative procedure described above was followed, except that reaction time was $36 \mathrm{~h}$. 4d was isolated as a light yellow oil. IR (neat, NaCl): 3395 (w), 3055 (w), 1759 (s), 1513 (s), 1602 (s), 1457 (m), 1249 (s), 1218 (s), 1193 (s), 1161 (s), 1123 (s), 1029 (m), 802 (m), 777 (m), 739 (m), $689(\mathrm{~m}) .{ }^{1} \mathrm{H} \mathrm{NMR}\left(\mathrm{CDCl}_{3}, 400 \mathrm{MHz}\right): \delta 8.14(1 \mathrm{H}, \mathrm{d}, J=8.1), 7.81(2 \mathrm{H}, \mathrm{dd}, J=7.3,6.8)$, $7.62(1 \mathrm{H}, \mathrm{d}, J=7.1), 7.51-7.35(5 \mathrm{H}, \mathrm{m}), 7.26-6.82(7 \mathrm{H}, \mathrm{m}), 5.13(1 \mathrm{H}, \mathrm{dd}, J=6.4,6.2), 5.00(1 \mathrm{H}$, bs), $3.87(3 \mathrm{H}, \mathrm{s}), 3.34(1 \mathrm{H}, \mathrm{dd}, J=15.2,6.4), 3.26(1 \mathrm{H}, \mathrm{dd}, J=15.2,6.4) .{ }^{13} \mathrm{C} \mathrm{NMR}\left(\mathrm{CDCl}_{3}\right.$, $100 \mathrm{MHz}): \delta 169.4,150.9,148.2$, 136.2, 133.6, 133.3, 130.8, 129.7, 129.1, 128.4, 127.1, 126.6, $126.5,126.3,125.4,121.9,121.5,120.5,118.9,113.0,110.3,93.4,82.0,55.8,43.9,41.3$. HRMS (ES) Calcd for $\mathrm{C}_{28} \mathrm{H}_{24} \mathrm{NO}_{3}$ : 422.1761; Found: $422.1756\left(\mathrm{M}+\mathrm{H}^{+}\right) \cdot[\alpha]_{\mathrm{D}}{ }^{20}=+63.2^{\circ}(c=$ $1.13, \mathrm{CHCl}_{3}$ ) for a $92 \%$ ee sample.

Optical purity of $\mathbf{4 d}$ was established by chiral HPLC analysis (OD column, 80/20 hexanes/ $i$ $\mathrm{PrOH}$ eluent, $1 \mathrm{~mL} / \mathrm{min}$, lamp setting of $254 \mathrm{~nm}, \mathrm{R}_{\mathrm{t}}($ major $)=28.66, \mathrm{R}_{\mathrm{t}}($ minor $\left.)=46.48\right)$.

(+)-3-(2-Methoxy-phenylamino)-undec-4-ynoic acid phenyl ester (4e). Isolated as a light yellow oil. IR (neat, $\mathrm{NaCl}$ ): 2930 (s), 2860 (m), 1765 (s), 1608 (s), 1513 (s), 1457 (s), 1249 (s),

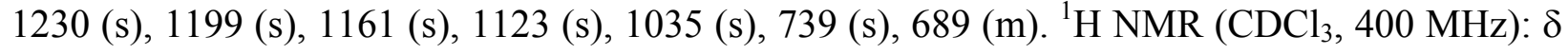
7.37 (2H, dd, $J=8.1,7.5), 7.22(1 \mathrm{H}, \mathrm{dd}, J=8.6,7.5), 7.08(2 \mathrm{H}, \mathrm{dd}, J=7.5,0.7), 6.92-6.73(4 \mathrm{H}$, m), $4.70(2 \mathrm{H}, \mathrm{bs}), 3.83(3 \mathrm{H}, \mathrm{s}), 3.10(1 \mathrm{H}, \mathrm{dd}, J=0.1,14.5), 3.00(1 \mathrm{H}, \mathrm{dd}, J=0.1,14.5), 2.16$ $(2 \mathrm{H}, \mathrm{dd}, J=7.0,7.0), 1.49-1.20(8 \mathrm{H}, \mathrm{m}), 0.86(3 \mathrm{H}, \mathrm{t}, J=6.6) .{ }^{13} \mathrm{C} \mathrm{NMR}\left(\mathrm{CDCl}_{3}, 100 \mathrm{MHz}\right): \delta$ 169.2 , 150.7, 147.7, 136.1, 129.5, 126.0, 121.7, 121.3, 118.1, 112.1, 110.0, 84.3, 79.0, 55.6, 42.8, 41.4, 31.5, 28.7, 28.5, 22.6, 18.8, 14.2. HRMS (ES) Calcd for $\mathrm{C}_{24} \mathrm{H}_{29} \mathrm{NO}_{3} \mathrm{Na}$ : 402.2039; Found: $402.2045(\mathrm{M}+\mathrm{Na}) .[\alpha]_{\mathrm{D}}{ }^{20}=+26.5^{\circ}\left(c=1.57, \mathrm{CHCl}_{3}\right)$ for an $86 \%$ ee sample.

Optical purity of $\mathbf{2 e}$ was established by chiral HPLC analysis (OD column, 95/5 hexanes $/ i \operatorname{PrOH}$ eluent, $1 \mathrm{~mL} / \mathrm{min}$, lamp setting of $254 \mathrm{~nm}, \mathrm{R}_{\mathrm{t}}($ minor $)=20.83, \mathrm{R}_{\mathrm{t}}($ major $\left.)=29.89\right)$. 
(+)-3-(2-Methoxy-phenylamino)-6-methyl-6-trimethylsilanyloxy-hept-4-ynoic acid phenyl ester (4f). The representative procedure described above was followed, except that the crude product was purified by silica gel washed with $1 \% \mathrm{Et}_{3} \mathrm{~N}$ and eluted with 99:1 hexane:Et ${ }_{2} \mathrm{O}$. 4f was isolated as a light yellow oil. IR (neat, NaCl): 3376 (br), 2980 (m), 2363 (m), 1759 (s), 1602 (m), 1507 (s), 1451 (m), 1249 (s), 1161 (s), 1123 (m), 1035 (s), 840 (s), 739 (m), 683 (m). ${ }^{1} \mathrm{H}$ NMR $\left(\mathrm{CDCl}_{3}, 400 \mathrm{MHz}\right): \delta$ 7.39-7.33 (2H, m), 7.25-7.20 (1H, m), 7.09-7.07 (2H, m), 6.9$6.73(4 \mathrm{H}, \mathrm{m}), 4.78-4.69(2 \mathrm{H}, \mathrm{m}), 3.82(3 \mathrm{H}, \mathrm{s}), 3.09(1 \mathrm{H}, \mathrm{dd}, J=6.2,15.0), 3.02(1 \mathrm{H}, \mathrm{dd}, J=6.2$, 15.0), $1.42(6 \mathrm{H}, \mathrm{d}, J=3.7), 0.1(9 \mathrm{H}, \mathrm{s}) .{ }^{13} \mathrm{C} \mathrm{NMR}\left(\mathrm{CDCl}_{3}, 100 \mathrm{MHz}\right): \delta 169.0,150.7,147.8$, 136.1, 129.6, 126.1, 126.0, 121.7, 121.3, 118.5, 112.4, 110.0, 88.7, 81.8, 66.6, 55.6, 42.9, 41.0, 33.2, 2.0. HRMS (ES) Calcd for $\mathrm{C}_{24} \mathrm{H}_{32} \mathrm{NO}_{4} \mathrm{Si}$ : 426.2101; Found: $426.2101\left(\mathrm{M}+\mathrm{H}^{+}\right) .[\alpha]_{\mathrm{D}}{ }^{20}=$ $+28.5^{\circ}\left(c=0.0135, \mathrm{CHCl}_{3}\right)$ for an $84 \%$ ee sample.

The optical purity of $\mathbf{4 f}$ was established by chiral HPLC analysis (OD column, 90/10 hexanes/ $i$-PrOH eluent, $1 \mathrm{~mL} / \mathrm{min}$, lamp setting of $230 \mathrm{~nm}, \mathrm{R}_{\mathrm{t}}($ major $)=9.75, \mathrm{R}_{\mathrm{t}}($ minor $\left.)=17.35\right)$.

(+)-5-Cyclohex-1-enyl-3-(2-methoxy-phenylamino)-pent-4-ynoic acid phenyl ester (4g). Isolated as a light yellow oil. IR (neat, $\mathrm{NaCl}$ ): 3389 (br), 2942 (m), 2873 (w), 1759 (s), 1602 (s), 1513 (s), 1463 (s), 1249 (s), 1224 (s), 1199 (s), 1167 (s), 1123 (s), 1029 (m), 746 (m), 683 (m). ${ }^{1} \mathrm{H} \mathrm{NMR}\left(\mathrm{CDCl}_{3}, 400 \mathrm{MHz}\right): \delta$ 7.40-7.35 (2H, m), 7.25-7.20 (1H, m), 7.10-7.08 (2H, m), 6.92$6.73(4 \mathrm{H}, \mathrm{m}), 6.06(1 \mathrm{H}, \mathrm{m}), 4.84(1 \mathrm{H}, \mathrm{dd}, J=6.6,6.6), 4.75(1 \mathrm{H}, \mathrm{bs}), 3.83(3 \mathrm{H}, \mathrm{s}), 3.13(1 \mathrm{H}, \mathrm{dd}$, $J=15.0,6.6), 3.11(1 \mathrm{H}, J=15.0,6.6), 2.07-2.05(4 \mathrm{H}, \mathrm{m}), 1.61-1.52(4 \mathrm{H}, \mathrm{m}) .{ }^{13} \mathrm{C} \mathrm{NMR}\left(\mathrm{CDCl}_{3}\right.$, $100 \mathrm{MHz}): \delta 169.1,150.7,147.7,136.0,135.4,129.5,126.1,121.7,121.3,120.2,118.2,112.1$, 110.0, 85.5, 85.3, 55.6, 43.2, 41.3, 29.3, 25.7, 22.3, 21.2. HRMS (ES) Calcd for $\mathrm{C}_{24} \mathrm{H}_{26} \mathrm{NO}_{3}$ : 376.1919; Found: $376.1913\left(\mathrm{M}+\mathrm{H}^{+}\right) .[\alpha]_{\mathrm{D}}{ }^{20}=+25.7^{\circ}\left(c=2.57, \mathrm{CHCl}_{3}\right)$ for a $90 \%$ ee sample.

Optical purity of $\mathbf{4 g}$ was established by chiral HPLC analysis (OD column, 90/10 hexanes/ $i$ PrOH eluent, $1 \mathrm{~mL} / \mathrm{min}$, lamp setting of $254 \mathrm{~nm}, \mathrm{R}_{\mathrm{t}}($ major $)=13.95, \mathrm{R}_{\mathrm{t}}($ minor $\left.)=20.00\right)$.

(+)-5-Cyclopropyl-3-(2-methoxy-phenylamino)-pent-4-ynoic acid phenyl ester (4h). Isolated as a light yellow oil. IR (neat, $\mathrm{NaCl}$ ): 3389 (w), 2250 (w), 1759 (s), 1602 (m), 1513 (s), 1451 (m), 1243 (s), 1218 (s), 1193 (s), 1167 (s), 1123 (s), 1029 (m), 739 (s), 683 (m). ${ }^{1} \mathrm{H}$ NMR $\left(\mathrm{CDCl}_{3}, 400 \mathrm{MHz}\right): \delta$ 7.39-7.34 $(2 \mathrm{H}, \mathrm{m}), 7.24-7.20(1 \mathrm{H}, \mathrm{m}), 7.10-7.07(2 \mathrm{H}, \mathrm{m}), 6.91-6.72(4 \mathrm{H}$, m), $4.67(2 \mathrm{H}, \mathrm{bs}), 3.83(3 \mathrm{H}, \mathrm{s}), 3.07(1 \mathrm{H}, \mathrm{dd}, J=14.8,6.0), 2.96(1 \mathrm{H}, \mathrm{dd}, J=15.0,6.1), 1.26-$ $1.18(1 \mathrm{H}, \mathrm{m}), 0.75-0.70(2 \mathrm{H}, \mathrm{m}), 0.64-0.60(2 \mathrm{H}, \mathrm{m}) .{ }^{13} \mathrm{C} \mathrm{NMR}\left(\mathrm{CDCl}_{3}, 100 \mathrm{MHz}\right): \delta 169.2$, $150.7,147.6,136.1,129.5,126.0,121.7,121.3,118.1,112.0,110.0,87.4,74.1,55.6,42.8,41.4$, 8.3, 8.2. HRMS (ES) Calcd for $\mathrm{C}_{21} \mathrm{H}_{22} \mathrm{NO}_{3}$ : 336.1589; Found: $336.1586\left(\mathrm{M}+\mathrm{H}^{+}\right) .[\alpha]_{\mathrm{D}}{ }^{20}=+47.6$ ${ }^{\circ}\left(c=2.00, \mathrm{CHCl}_{3}\right)$ for a $90 \%$ ee sample.

Optical purity of $\mathbf{4 h}$ was established by chiral HPLC analysis (OD column, 90/10 hexanes/ $i$ PrOH eluent, $1 \mathrm{~mL} / \mathrm{min}$, lamp setting of $220 \mathrm{~nm}, \mathrm{R}_{\mathrm{t}}($ minor $)=18.99, \mathrm{R}_{\mathrm{t}}($ major $\left.)=21.74\right)$. 
(-)-3-(2-Methoxy-phenylamino)-5-naphthalen-1-yl-pentanoic acid phenyl ester (5). To a solution of $4 \mathbf{d}$ (100 $\mathrm{mg}$ of a $92 \%$ ee sample, $0.24 \mathrm{mmol})$ in $\mathrm{MeOH}(15 \mathrm{~mL})$ was added $50.5 \mathrm{mg}$ $\mathrm{Pd}(\mathrm{C})(20 \mathrm{~mol} \% \mathrm{Pd}, 10 \% \mathrm{Pd}(\mathrm{C}))$. The headspace of the solution was purged with $\mathrm{H}_{2}$ and the mixture was allowed to stir at $23{ }^{\circ} \mathrm{C}$ under 1 atmosphere of $\mathrm{H}_{2}$ (balloon) for $3 \mathrm{~h}$ after which time the solution was filtered through celite, which was subsequently washed with $\mathrm{MeOH}(3 \times 10 \mathrm{~mL})$. Removal of the volatiles and purification of the resulting residue by silica gel column chromatography (9:1 hexanes: $\left.\mathrm{Et}_{2} \mathrm{O}\right)$ provided $85 \mathrm{mg}(0.20 \mathrm{mmol} ; 84 \%$ yield) of 5. IR (neat, $\mathrm{NaCl}$ ): 3412 (br), 2940 (w), 1752 (s), 1608 (m), 1510 (s), 1464 (m), 1250 (m), 1221 (s), 1193 (s), 1164 (m), 1123 (m), 1031 (m), 783 (m), 737 (s), 691 (w). ${ }^{1} \mathrm{H}$ NMR (CDCl, $\left.400 \mathrm{MHz}\right): \delta 8.03$ (1H, dd, $J=4.0,2.9), 7.86(1 \mathrm{H}, \mathrm{dd}, J=5.7,4.0), 7.73(1 \mathrm{H}, \mathrm{dd}, J=8.0,8.0), 7.49-7.18(7 \mathrm{H}, \mathrm{m})$, 6.91-6.71 (6H, m), 4.51 (1H, bs), 4.10 (1H, bs), $3.88(3 \mathrm{H}, \mathrm{s}), 3.39-3.19(2 \mathrm{H}, \mathrm{m}), 2.92(1 \mathrm{H}, \mathrm{dd}, J$ $=14.8,5.7), 2.77(1 \mathrm{H}, \mathrm{dd}, J=14.8,5.7), 2.28-2.08(2 \mathrm{H}, \mathrm{m}) \cdot{ }^{13} \mathrm{C} \mathrm{NMR}\left(\mathrm{CDCl}_{3}, 100 \mathrm{MHz}\right): \delta$ $170.4,150.6,147.3,137.8,137.0,134.1,131.9,129.5,129.0,127.0,126.3,126.1,126.0,125.7$, 125.6, 123.8, 121.7, 121.6, 117.1, 110.9, 110.0, 55.6, 50.3, 40.1, 36.5, 29.7. HRMS (ES) Calcd for $\mathrm{C}_{28} \mathrm{H}_{28} \mathrm{NO}_{3}$ : 426.2076; Found: $426.2069\left(\mathrm{M}+\mathrm{H}^{+}\right) .[\alpha]_{\mathrm{D}}{ }^{20}=-10.7^{\mathrm{o}}\left(c=2.74, \mathrm{CHCl}_{3}\right)$ for a $92 \%$ ee sample.

(+)-3-tert-Butoxycarbonylamino-5-phenyl-pent-4-ynoic acid phenyl ester (6). To a solution of $\mathrm{PhI}(\mathrm{OAc})_{2}(0.70 \mathrm{~g}, 2.1 \mathrm{mmol})$ in $\mathrm{MeOH}(10 \mathrm{~mL})$ and $\mathrm{AcOH}(0.5 \mathrm{~mL})$ at $22{ }^{\circ} \mathrm{C}$ was added a solution of $\mathbf{4 b}(0.20 \mathrm{~g}, 0.54 \mathrm{mmol})$ in $\mathrm{MeOH}(2 \mathrm{~mL})$ slowly at $22{ }^{\circ} \mathrm{C}$ over $30 \mathrm{~min}$. The mixture was then allowed to stir at $22{ }^{\circ} \mathrm{C}$ for an additional $30 \mathrm{~min}$ upon which time $5 \mathrm{~mL}$ of a $10 \%$ aqueous solution of $\mathrm{HCl}$ was added. The mixture was allowed to stir for $30 \mathrm{~min}$ at which time $5 \mathrm{~mL}$ of a $20 \%$ aqueous solution of $\mathrm{Na}_{2} \mathrm{~S}_{2} \mathrm{O}_{3}$ was added and stirring was allowed to continue for an additional $30 \mathrm{~min}$. The solution was made basic by the addition of $\mathrm{Na}_{2} \mathrm{CO}_{3}(\mathrm{~s})$ (solution turned dark red) and $\mathrm{Boc}_{2} \mathrm{O}(0.46 \mathrm{~g}, 2.1 \mathrm{mmol})$ was added followed by $\mathrm{CH}_{2} \mathrm{Cl}_{2}(5 \mathrm{~mL})$. The mixture was allowed to stir at $22{ }^{\circ} \mathrm{C}$ for $12 \mathrm{~h}$. The layers were separated and the aqueous layer was washed with $\mathrm{CH}_{2} \mathrm{Cl}_{2}(2 \times 25 \mathrm{~mL})$. The combined organic layers were washed with brine $(1 \times 10 \mathrm{~mL})$, dried over $\mathrm{MgSO}_{4}$ and volatiles were removed in vacuo. The desired product was recrystallized from hot hexanes to afford $\mathbf{6}(137 \mathrm{mg}, 0.37 \mathrm{mmol}, 68 \%$ yield $)$ as a white solid. IR (neat, $\mathrm{NaCl}$ ): 3345 (br), 2986 (w), 1759 (s), 1715 (s), 1495 (s), 1369 (m), 1193 (m), 1161 (s), $1023(\mathrm{w}), 765$ (m), 689 (m). ${ }^{1} \mathrm{H}$ NMR $\left(\mathrm{CDCl}_{3}, 400 \mathrm{MHz}\right): \delta 7.43-7.08$ (10H, m), 5.37 (1H, bs), $5.16(1 \mathrm{H}, \mathrm{bs}), 3.95(1 \mathrm{H}, \mathrm{dd}, J=15.6,5.5), 3.00(1 \mathrm{H}, \mathrm{dd}, J=15.6,5.6), 1.47(9 \mathrm{H}, \mathrm{s}) .{ }^{13} \mathrm{C}$ NMR $\left(\mathrm{CDCl}_{3}, 100 \mathrm{MHz}\right): \delta 168.9,154.8,150.6,131.9,129.6,128.7,128.4,126.2,122.4,121.7,86.9$, 83.8, 80.4, 41.1, 40.3, 28.5. HRMS (ES) Calcd for $\mathrm{C}_{22} \mathrm{H}_{23} \mathrm{NO}_{4} \mathrm{Na}$ : 388.1524; Found: 388.1525 $\left(\mathrm{M}+\mathrm{Na}^{+}\right) \cdot[\alpha]_{\mathrm{D}}{ }^{20}=+45.6^{\circ}\left(c=1.64, \mathrm{CHCl}_{3}\right)$ for a $92 \%$ ee sample.

(+)-3-(2-Benzyloxycarbonylamino-3-methylbutyrylamino)-5-phenylpent-4-ynoic acid tert-butyl ester (10). To a solution of $\mathrm{PhI}(\mathrm{OAc})_{2}(0.50 \mathrm{~g}, 1.6 \mathrm{mmol})$ in $\mathrm{MeOH}(15 \mathrm{~mL})$ and $\mathrm{AcOH}(0.1 \mathrm{~mL})$ at $22{ }^{\circ} \mathrm{C}$ was added a solution of $\mathbf{4 b}(0.13 \mathrm{~g}, 0.35 \mathrm{mmol})$ in $\mathrm{MeOH}(2 \mathrm{~mL})$ 
slowly at $22{ }^{\circ} \mathrm{C}$ over $30 \mathrm{~min}$. The mixture was then allowed to stir at $22{ }^{\circ} \mathrm{C}$ for an additional 30 min upon which time $5 \mathrm{~mL}$ of a $10 \%$ aq. $\mathrm{HCl}$ solution was added. The mixture was allowed to stir for 30 minutes at which time $5 \mathrm{~mL}$ of a $20 \%$ aqueous solution of $\mathrm{Na}_{2} \mathrm{~S}_{2} \mathrm{O}_{3}$ was added and stirring was continued for an additional $30 \mathrm{~min}$. The resulting mixture was made basic by the addition of $\mathrm{Na}_{2} \mathrm{CO}_{3}(\mathrm{~s})$ (solution turned dark red). Cbz-Val-OSu $(0.85 \mathrm{~g}, 2.4 \mathrm{mmol})$ was added, followed by $\mathrm{CH}_{2} \mathrm{Cl}_{2}(5 \mathrm{~mL})$. The mixture was allowed to stir at $22{ }^{\circ} \mathrm{C}$ for $12 \mathrm{~h}$. The layers were then separated and the aqueous layer was washed with $\mathrm{CH}_{2} \mathrm{Cl}_{2}(2 \times 25 \mathrm{~mL})$. The combined organic layers were washed with brine $(1 \times 10 \mathrm{~mL})$, dried over $\mathrm{MgSO}_{4}$ and volatiles were removed in vacuo. The resulting residue was purified by silica gel chromatography $3: 2$ EtOAc:hexanes) to afford $\mathbf{1 0}$ (127 mg, $0.26 \mathrm{mmol}, 73 \%$ yield) as a white solid. IR (neat, $\mathrm{NaCl}$ ): 3307 (br), 2986 (w), 2980 (m), 1727 (s), 1526 (s), 1369 (m), 1249 (m), 1155 (s), 1029 (w), 752 (m), $689(\mathrm{~m}) .{ }^{1} \mathrm{H}$ NMR $\left(\mathrm{CDCl}_{3}, 400 \mathrm{MHz}\right): \delta 7.37-7.24(10 \mathrm{H}, \mathrm{m}), 6.93(1 \mathrm{H}, \mathrm{d}, J=8.6), 5.41$ $(1 \mathrm{H}, \mathrm{dd}, J=8.6,9.1), 5.28-5.23(1 \mathrm{H}, \mathrm{m}), 5.10(2 \mathrm{H}, \mathrm{s}) 4.07-4.00(1 \mathrm{H}, \mathrm{m}), 2.73(1 \mathrm{H}, \mathrm{dd}, J=15.8$, 4.8), $2.61(1 \mathrm{H}, \mathrm{dd}, J=15.8,4.9), 2.17-2.10(1 \mathrm{H}, \mathrm{m}), 1.45(9 \mathrm{H}, \mathrm{s}), 0.99-0.90(6 \mathrm{H}, \mathrm{m}) .{ }^{13} \mathrm{C} \mathrm{NMR}$ $\left(\mathrm{CDCl}_{3}, 100 \mathrm{MHz}\right): \delta 170.1,169.8,169.7,131.9,131.8,128.6,128.5,128.5,128.3,128.2,128.2$, 128.1, 128.1, 81.8, 81.7, 40.8, 38.7, 38.6, 28.2, 19.2, 19.1. HRMS (ES) Calcd for $\mathrm{C}_{28} \mathrm{H}_{34} \mathrm{~N}_{2} \mathrm{O}_{5} \mathrm{Na}$ : 501.2365; Found: $501.2368(\mathrm{M}+\mathrm{Na})$. $[\alpha]_{\mathrm{D}}{ }^{20}=+0.2^{\circ}\left(c=0.93, \mathrm{CHCl}_{3}\right)$ for a $>98 \%$ ee sample. 